\title{
MULTILAYER MODELLING OF STAINLESS STEEL WITH NANOCRYSTALLIZED SUPERFICIAL LAYER
}

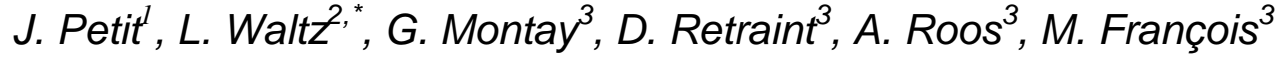 \\ ${ }^{1}$ Laboratoire des Procédés et Ingénierie en Mécanique et Matériaux (PIMM), Arts et Métiers \\ ParisTech, 75013 Paris, FRANCE \\ ${ }^{2}$ Laboratoire de Mécanique et Génie Civil de Montpellier (LMGC), University of Montpellier II, \\ place Eugène Bataillon, 34000 Montpellier, FRANCE \\ ${ }^{3}$ Institut Charles Delaunay - LASMIS, UMR CNRS 6279, University of Technology of \\ Troyes, 10010 Troyes, FRANCE
}

\begin{abstract}
*Corresponding author. Present address: University of Montpellier II, Montpellier, France. E-mail address: laurent.waltz@univ-montp2.fr

Tel.: $33(0) 4$ 67.14.39.23
\end{abstract}

\begin{abstract}
In order to obtain the macroscopic mechanical response of a $316 \mathrm{~L}$ stainless steel, nanocrystallized by a SMAT treatment, a multilayer model is proposed. The constitutive behaviour of each layer is determined from tensile tests or by an inverse method and its thickness is evaluated from SEM and TEM micrographic analyses and local hardness measurements. The consistency of the model is verified by its ability to predict the strain at which diffuse necking occurs.
\end{abstract}

\section{Key Words:}

Nanocrystalline Materials; SMAT; Localization; Electronic Speckle Pattern Interferometry (ESPI); Tensile Test; Modelling. 


\section{Introduction}

Ultrafine grain materials with mean grain size smaller than $100 \mathrm{~nm}$ are actively studied because of their enhanced mechanical properties, such as high strength, hardness and superplasticity. In order to obtain an ultrafine grain structure in metallic materials and alloys, several authors have used the Surface Mechanical Attrition Treatment (SMAT) which is based on severe plastic deformation. In the surface layer (several tens of micrometers thick) of bulk materials, this treatment induces grain refinement, sometimes down to the nanometric scale [1-5]. As a result of plastic deformation of the surface layer, the micrometric grains are refined without changing chemical composition and without creating any porosity. Unfortunately, the ductility of nanocrystallized materials significantly decreases compared with their traditional coarse-grained counterparts [6-8]. The multilayered structure of SMATed components allows retaining a ductility that reaches $15 \%$ to $20 \%$ total strain while increasing the overall strength. It is thus important to investigate the mechanical behaviour of each layer in detail and how their interactions play a role in the ductility of the component.

In the present paper, a multilayer model for the macroscopic mechanical behaviour of a SMATed 316L plate is presented. It takes as input the mechanical behaviour of each layer and its proportion in the whole material. The mechanical behaviour of each layer is directly fitted to tensile curves, except for the most refined layer, where it is obtained by an inverse method. The average thickness of each layer is determined by Scanning Electron Microscopy (SEM) and a hardness profile, obtained by nanoindentation along the cross-section. Moreover, the model also predicts the deformation corresponding to diffuse necking, i.e. when the maximum value of the tensile force is reached, by applying Considère's criterion [9] to an uniaxial tensile test (see §3.3).

\section{Specimens and identification of the parameters of the constitutive law}

\subsection{Layers and model description}

The material used in this investigation is a commercial $316 \mathrm{~L}$ face-centred cubic austenitic stainless steel. For experimental tests, $19 \times 3.6 \times 1 \mathrm{~mm}^{3}$ plates were used, with chemical 
composition as given in Table 1. The initial microstructure of the as-received material contains grains with diameters ranging between 40 and $120 \mu \mathrm{m}$. The two nanocrystallized surfaces of this sheet were obtained by 30 minutes of ultrasonic-assisted SMAT in air and at room temperature, with $3 \mathrm{~mm}$ diameter stainless steel shot and a vibration frequency of 20 $\mathrm{kHz}$. A more detailed description of the SMAT process can be found in [1] and [4].

In the model, three distinct layers are distinguished [5, 10-12]:

- the nanocrystalline layer at the material surface with grain size less than $100 \mathrm{~nm}$,

- the hardened transition layer with a gradient of grain size and a high density of mechanical twins,

- the coarse grained raw material layer in the middle of the sample.

Each layer has its own elastoplastic behaviour, assumed to follow a power law. As the plate is SMATed on both sides, the model is divided into five layers (see Fig. 1): 2 nanocrystalline layers, 2 transition layers and 1 core material layer at the middle of the plate. The general scheme synthesizing the approach to recover the macroscopic behaviour of the SMATed sample is given in Fig. 2 and explained afterwards.

To obtain the constitutive behaviour of the three layers, three specimens were required:

- one for the core material: as it can be assumed that SMAT affects only the surface of the structure, a specimen was machined in the as-received material,

- one for the transition layer: the specimen was obtained by mechanical polishing to remove the nanocrystallized layer and the core layer,

- one for the nanocrystalline layer: as this layer is too thin to be isolated and to be handled with the required accuracy, a composite specimen containing both the nanocrystalline and the transition layer was manufactured by mechanical polishing from a SMATed specimen. The behaviour was then obtained indirectly through an inverse method, as explained below.

\subsection{Experiments}

Tensile tests were carried out at room temperature on a screw driven Kammrath \& Weiss micro-tensile machine at a fixed strain rate of $2 \cdot 10^{-4} \mathrm{~s}^{-1}$. The dimensions of the tensile 
specimens were $36 \mathrm{~mm}$ total length, with a gauge length of $19 \mathrm{~mm}$ and an average crosssection of $e \times 3.6 \mathrm{~mm}^{2}$, where ' $e$ ' is the thickness of the sample.

After tensile tests and fitting to the experimental curves by a Least Squares error minimization resolved by the Levenberg-Marquardt algorithm, the following parameters were obtained:

\section{Core material:}

$$
\sigma_{c}=\sigma_{0 c}+K_{c} \cdot \varepsilon^{n_{c}}=290+1255 \cdot \varepsilon^{0,733}
$$

Transition layer:

$$
\sigma_{t}=\sigma_{0 t}+K_{t} \cdot \varepsilon^{n_{t}}=730+481 \cdot \varepsilon^{0,502}
$$

with $\varepsilon$ the plastic strain along the tensile axis, the index $c$ denoting 'core material' and the index $t$ denoting 'transition layer', and $\sigma_{0 c}, \sigma_{0 t}, K_{c}, K_{t}, n_{c}$ and $n_{t}$ material parameters: $\sigma_{0 c}$ and $\sigma_{0 t}$ represent the yield stress; $K_{c}, K_{t}, n_{c}$ and $n_{t}$ are hardening coefficients. Fig. 3 shows the true stress - strain curves for the as-received coarse grain material and the transition layer, with their corresponding models.

\subsection{Inverse method}

Knowing the two constitutive behaviours from tensile tests, the behaviour of the nanocrystalline layer can be obtained. From the tensile test, the behaviour of the nanocrystalline + transition layer is given by:

Nanocristalline + transition layer:

$$
\sigma_{n+t}=\sigma_{0 n+t}+K_{n+t} \cdot \varepsilon^{n_{n+t}}=810+400 \cdot \varepsilon^{0,391}
$$

with $\sigma_{0 n+t}, K_{n+t}$, and $n_{n+t}$, the material parameters, analogous to the ones in the previous section.

The thickness of the nanocristalline layer is $15 \mu \mathrm{m}( \pm 2 \mu \mathrm{m})$ according to scanning electron microscopy (SEM) observations (not shown here). The thickness of the nanocristalline + transition layer, $e_{n+t}$, is determined from local hardness measurements obtained by 
nanoindentation tests along the sample depth [12]. It is estimated at $245 \mu \mathrm{m}( \pm 10 \mu \mathrm{m})$, when the local hardness, which decreases with distance from the surface, is stabilized. Consequently, the thickness of the transition layer becomes $230 \mu \mathrm{m}( \pm 10 \mu \mathrm{m})$.

The nanocrystalline layer behaviour is also assumed to follow a power law. By considering that plane sections remain plane during the deformation (verified §3.2) and imposing global equilibrium, the relationship

$$
\sigma_{n+t}=\frac{S_{n}}{S_{n+t}} \sigma_{n}+\frac{S_{t}}{S_{n+t}} \sigma_{t}=\frac{e_{n}}{e_{n+t}} \sigma_{n}+\frac{e_{t}}{e_{n+t}} \sigma_{t},
$$

gives the total stress of a specimen only composed of the nanocrystalline + transition layer, with $\sigma_{n}$ the stress in the nanocrystalline layer. Thus, the objective function, $f_{o b j}$, to be minimized in the least-square sense in order to determine the parameters of $\sigma_{n}$, can be built:

$$
f_{o b j}=\sqrt{\frac{1}{N} \sum_{i=1}^{N}\left(\sigma_{n+t}^{i}-\left[\frac{e_{n}}{e_{n+t}} \sigma_{n}^{i}+\frac{e_{t}}{e_{n+t}} \sigma_{t}^{i}\right]\right)^{2}},
$$

where $N$ is the number of points, arbitrarily chosen but large enough, describing each true stress - true strain curve and $\sigma_{n+t}$ and $\sigma_{t}$ determined experimentally. The resolution of equation (1.5) is an optimization problem which was treated with a Matlab program. The algorithm seeks the minimum of a nonlinear multivariable function with constraints by using the Sequential Quadratic Programmation (SQP) method. Constraints of the present problem are only the domain boundaries of the parameters. After optimization, the following result is obtained:

Nanocrystalline layer:

$$
\sigma_{n}=\sigma_{0 n}+K_{n} \cdot \varepsilon^{n_{n}}=1757+338 \cdot \varepsilon^{0,0712}
$$

with $\sigma_{0 n}, K_{n}$ and $n_{n}$ material parameters. Local minima were avoided by rerunning the optimization with many different initial parameters. 


\section{Results and discussion}

\subsection{Obtained values}

The obtained hardening exponent $n_{n}$ is consistent with the result given by Chen et al. [8], who have also studied a $316 \mathrm{~L}$ stainless steel treated by SMAT in the same conditions as in the present paper. The yield stress $(0.2 \%$ offset $)$ which reaches $\approx 1950 \mathrm{MPa}$ in the present work is appreciably higher than theirs $(\approx 1455 \mathrm{MPa})$. The discrepancy can be explained by several phenomena:

- Firstly, in [8], difficulties associated to sample preparation by mechanical polishing with an average thickness of $15 \mu \mathrm{m}$ lead to heterogeneities and defects in the cross section of the sample. Thus, during tensile tests, the sample will be torn apart prematurely due to early localization.

- Secondly, over- or underestimation of the thicknesses of the different layers by micrographic means can lead to a difference of the yield stress of several tens of MPa. Besides, the measurement uncertainties associated to the load cell are estimated at approximately $50 \mathrm{MPa}$. In addition, considering only weighted average thicknesses in our reasoning and not taking into account any interaction between the nanocrystalline layer and the transition layer can appreciably modify the stress value. After sensitivity study, by varying the thickness of each layer within their respective uncertainty range and the obtained materials parameters (for the layers ' $t$ ' and ' $n+t$ ') in a $5 \%$ error range, an error of about $8 \%$ is estimated on the behaviour $\sigma_{n}$ of the nanocrystalline layer. By applying the ISO 07-020 procedure [13], these 8\% added to the uncertainties due to the load cell precision lead to an uncertainty of near $8.5 \%$, which doesn't explain alone the gap between our value of yield stress and the Chen's one.

\subsection{Plane sections assumption}

In order to justify the use of equation (1.4) as starting point for our model, the hypothesis that plane sections remain plane was verified by measuring the deformation through the thickness of the sample at one edge with Electronic Speckle Pattern Interferometry (ESPI) [14-17]. This technique allows very accurate measurements of displacement fields with a resolution of about $20 \mathrm{~nm}$. Fringes (isovalues of gray level) can be interpreted as lines of isodisplacements. The displacement component is measured parallel to the sensitivity vector, which is horizontal 
in the images of Fig. 4. Only regularly spaced and straight fringes along the length of the specimen can be seen. This indicates that, despite heterogeneity in constitutive behaviour, the displacement field is constant along the thickness of the specimen, at least at the free surface. Fig. 4 shows the fringe patterns at different stages during tensile test before necking occurs.

\subsection{Global behaviour and necking prediction}

The global behaviour of the multilayered structure was obtained by considering again that plane sections remain plane during the deformation. The macroscopic stress for the multilayer model of a 316L stainless steel treated by SMAT can then be expressed as:

$$
\sigma_{\text {tot }}=\frac{2 \sigma_{n} \cdot S_{n}+2 \sigma_{t} \cdot S_{t}+\sigma_{c} \cdot S_{c}}{S_{t o t}}=\frac{2 \sigma_{n} \cdot e_{n}+2 \sigma_{t} \cdot e_{t}+\sigma_{c} \cdot e_{c}}{e_{t o t}},
$$

with $S_{n}, e_{n}$ : section and thickness of the nanocristalline layer

$S_{t}, e_{t}:$ section and thickness of the transition layer

$S_{c}, e_{c}$ : section and thickness of the core material

$S_{t o t}, e_{t o t}:$ total section and total thickness

As noted previously, the section ratio is equal to the thickness ratio due to the same width of all the layers.

Fig. 5 displays the true stress - true strain curves for the SMATed material obtained experimentally and by the multilayer model. The two curves superimpose along a large part of the plastic domain. They part after the diffuse necking and there is a small difference during the elastoplastic transition, because modelling the material behaviour after the diffuse neck would require at least one damage parameter and the complex geometry would need a structure calculation. Also, using a model with only three distinct layers with uniform behaviour cannot properly describe the progressive yield of the transition layer.

To test the ability of our model to describe the elastoplastic behaviour of SMATed structures, it was used to predict the onset of diffuse necking with the help of Considère's criterion. This criterion [9] stipulates that the diffuse neck starts when the maximum force applied during a tensile test is reached, that is:

$$
\frac{d F}{d \varepsilon}=0,
$$


where $F$ is the tensile force and $\varepsilon$ represents the plastic strain along the tensile axis (elasticity is neglected at this stage). This is a point of plastic instability. Before this point, to get a deformation increment, the force has to be increased. From this point on, the deformation keeps rising while the force decreases: any new imposed deformation can not be compensated by the hardening anymore.

The total force $F$, by considering that plane sections remain plane during the deformation, can be expressed as:

$$
F=2 \sigma_{n} \cdot S_{n}+2 \sigma_{t} \cdot S_{t}+\sigma_{c} \cdot S_{c}
$$

Consequently, Considère's criterion becomes:

$$
\begin{aligned}
\frac{d F}{d \varepsilon}= & 2 S_{n} \frac{d \sigma_{n}}{d \varepsilon}+2 \sigma_{n} \frac{d S_{n}}{d \varepsilon}+2 S_{t} \frac{d \sigma_{t}}{d \varepsilon}+2 \sigma_{t} \frac{d S_{t}}{d \varepsilon}+S_{c} \frac{d \sigma_{c}}{d \varepsilon}+\sigma_{c} \frac{d S_{c}}{d \varepsilon} \\
= & 2 S_{n} \cdot n_{n} \cdot K_{n} \cdot \varepsilon^{n_{n}-1}-2 S_{n} \cdot K_{n} \cdot \varepsilon^{n_{n}}-2 \sigma_{0 n} \cdot S_{n}+2 S_{t} \cdot n_{t} \cdot K_{t} \cdot \varepsilon^{n_{t}-1}-2 S_{t} \cdot K_{t} \cdot \varepsilon^{n_{t}} \\
& -2 \sigma_{0 t} \cdot S_{t}+S_{c} \cdot n_{c} \cdot K_{c} \cdot \varepsilon^{n_{c}-1}-S_{c} \cdot K_{c} \cdot \varepsilon^{n_{c}}-\sigma_{0 c} \cdot S_{c} \\
= & 0 .
\end{aligned}
$$

In this equation, each surface $S_{i}$, where $i=n, t$ or $c$, is expressed in the following way: $S_{i}=S_{i 0} \cdot e^{-\varepsilon}$ where $S_{i 0}$ is the initial surface of each layer. This expression is obtained by assuming plastic incompressibility and by neglecting elasticity.

There is no analytical solution to this equation. It was thus solved numerically by Newton's method. The obtained solution, with all parameters previously determined, is $\varepsilon=25.8 \%$. Experimentally, the onset of the diffuse necking was found to be (see Fig. 5) $\varepsilon=25.6 \%$. It can be seen that the two values are very close, which means that the model is able to predict the onset of diffuse necking.

\subsection{Ductility of the nanocrystalline layer}

By applying Considère's criterion, the elongation-to-localization of the nanocrystalline layer is found to be $1.8 \%$. Even though this value is half of the one obtained by Chen, it is consistent with a brittle behaviour predicted for nanostructured materials [18]. Also, the 
deformation mechanisms in nanostructured materials are not well known yet and the application of Considère's criterion might be inadequate for this kind of material if the hardening mechanisms are not predominant. Moreover, it can be observed that, due to the multilayer structure in the SMATed sample, the global localization appears at $25.6 \%$, which is much later than $1.8 \%$. This justifies the fact that an interaction exists between the nanocrystalline layer and the transition layer and also between the transition layer and the core material.

\subsection{Stresses in the transverse direction}

Another assumption used in the model is that each layer is free to deform in the transverse direction with a transverse stress that remains zero. This may not always be the case, in particular during the elastoplastic transition where some layers are still elastic while others are already plastic. A finite element simulation was performed without enforcing this assumption, and it was found that it does not affect significantly the results.

\subsection{Residual stresses after SMAT}

SMAT causes a plastic strain in the superficial layers of the treated specimens. The resulting incompatibility leads to a residual stress gradient along the depth. In turn, these residual stresses will shift the observed yield stress of the material. In the present paper, this effect was neglected, or, in other words, the yield stresses that were determined are effective values integrating residual stresses.

\section{Conclusion}

The purely uniaxial multilayer model proposed in the present paper is very simple. However it allows an accurate description of the tensile elastoplastic behaviour of a SMATed specimen from the yield point to the onset of diffuse necking. It relies on the assumption that strains are homogeneous along the thickness, assumption which is supported by measurements carried out using Electronic Speckle Pattern Interferometry. This model is also able to evaluate, in an accurate way (approximately $0.5 \%$ of error), the deformation satisfying Considère's criterion. 
The model was used in an inverse method to determine the constitutive behaviour of the nanocrystalline layer of a SMATed 316L steel. The following results were obtained:

- the yield stress is $1950 \mathrm{MPa}$, which is about seven times higher than that of a coarse grain sample,

- the hardening coefficient of its constitutive power law is low and equal to 0.0712 ,

- the multiplier coefficient in front of the hardening term is equal to 338,

- the necking of the isolated nanocrystalline layer should occur at a strain of about $1.8 \%$, however due to the presence of the transition and core layers, the global necking occurs much later at $25.6 \%$ strain.

In order to better describe the experimental curve at the elastoplastic transition, a continuous model will be developed in future work where the behaviour of each elementary layer will be expressed in function of the thickness. Residual stresses will be measured and taken into account in the model to obtain the intrinsic yield stress of each layer.

\section{Acknowledgements}

The authors gratefully acknowledge the Champagne-Ardenne region as well as Europe for financial support.

\section{References}

[1] Lu K, Lu J, Surface nanocrystallization (SNC) of metallic materials-presentation of the concept behind a new approach. Journal of Materials Science and Technology 1999;15:193-197.

[2] Tao NR, Wang ZB, Tong WP, Sui ML, Lu J, Lu K, An investigation of surface nanocrystallization mechanism in Fe induced by surface mechanical attrition treatment. Acta Materiala 2002;50:4603-4616.

[3] Tong WP, Tao NR, Wang ZB, Lu J, Lu K, Nitriding iron at lower temperatures. Science 2003;299:686-688. 
[4] Lu K, Lu J, Nanostructured surface layer on metallic materials induced by surface mechanical attrition treatment. Materials Science and Engineering A 2004;375:38-45.

[5] Waltz L, Retraint D, Roos A, Olier P, Combination of surface nanocrystallization and co-rolling: Creating multilayer nanocrystalline composites. Scripta Materiala 2009;60:21-24. (Waltz et al., 2009)

[6] Sanders PG, Eastman JA, Weertman JR, Elastic and tensile behavior of nanocrystalline copper and palladium. Acta Materialia 1997;45:4019-4025.

[7] Jia D, Ramesh KT, Ma E, Failure mode and dynamic behavior of nanophase iron under compression. Scripta Materiala 1999;42:73-78.

[8] Chen XH, Lu J, Lu L, Lu K, Tensile properties of nanocrystalline 316L austenitic stainless steel. Scripta Materiala 2005;52:1039-1044.

[9] Considère A, Mémoire sur l'Emploi du Fer et de l'Acier dans les Constructions. Annales des Ponts et Chaussées 1885;9:574-775.

[10] Roland T, Retraint D, Lu J, Lu K, Generation of nanostructures on 316L stainless and its effect on mechanical behaviour. Materials Science Forum 2005;490-491:625-630.

[11] Roland T, Retraint D, Lu J, Lu K, Enhanced mechanical behavior of a nanocristallised stainless steel and its thermal stability. Materials Science and Engineering A 2007;445446:281-288.

[12] Waltz L, Retraint D, Roos A, Olier P, Lu J, High strength nanocrystallized multilayered structure obtained by SMAT and co-rolling. Materials Science Forum 2009;614:249254. (Waltz et al., 2009)

[13] Guide for the expression of Uncertainty of Measurement (GUM), ISO 07-020,1995.

[14] Cloud G. Optical Methods of Engineering Analysis, Cambridge University Press, NY, 1995.

[15] Jones R, Wykes C. Holographic and Speckle Interferometry, vol. 6, Cambridge Studies in Modern Optics, second ed., Cambridge University Press, Cambridge, 1989.

[16] Guelorget B, Francois M, Vial-Edwards C, Montay G. Strain rate measurement by ESPI: A new look at the strain localization onset. Materials Science and Engineering A 2006;415:234-241. 
[17] Petit J, Montay G, François M, Localization phenomenon investigation on SMATed stainless steel samples by Speckle Interferometry. Strain 2010;DOI:10.1111/j.14751305.2009.00726.x.

[18] Wang YM, Wang K, Pan D, Lu K, Hemker KJ, Ma E, Microsample tensile testing of nanocrystalline copper. Scripta Materialia 2003;48:1581-1586. 


\begin{tabular}{|c|c|c|c|c|c|c|c|c|c|c|c|c|}
\hline Elements & $\mathbf{C}$ & $\mathbf{S i}$ & $\mathbf{M n}$ & $\mathbf{P}$ & $\mathbf{S}$ & $\mathbf{C r}$ & $\mathbf{M o}$ & $\mathbf{N i}$ & $\mathbf{N}$ & $\mathbf{C u}$ & $\mathbf{B}$ & $\mathbf{C o}$ \\
\hline \% Weight & 0,025 & 0,38 & 1,33 & 0,027 & 0,002 & 16,70 & 2,09 & 10,20 & 0,03 & 0,40 & 0,0006 & 0,07 \\
\hline
\end{tabular}

Table 1: Nominal chemical composition of the 316L stainless steel used in this work. 


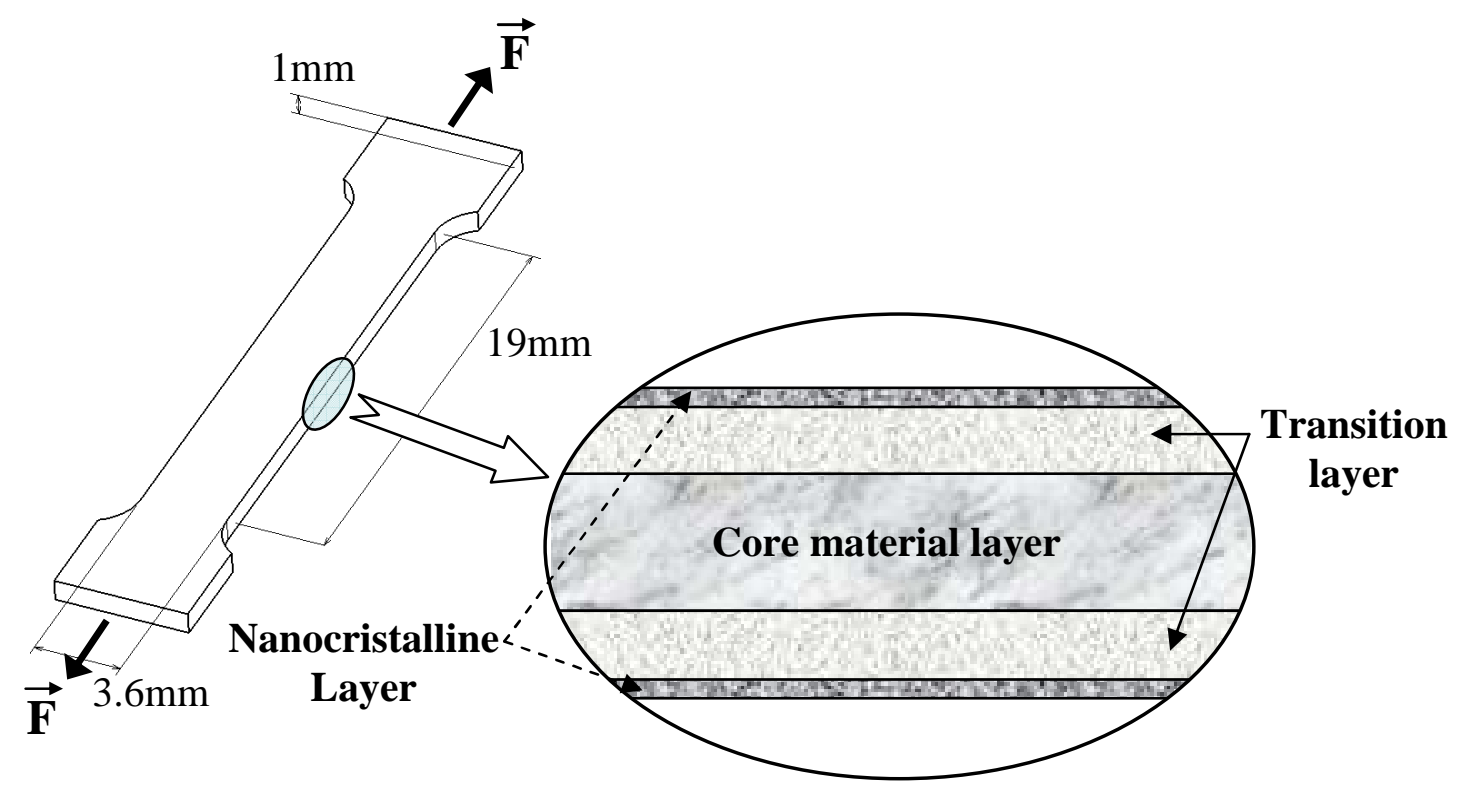

Fig. 1: Multilayer model showing the cross-section of a sample treated by SMAT. 


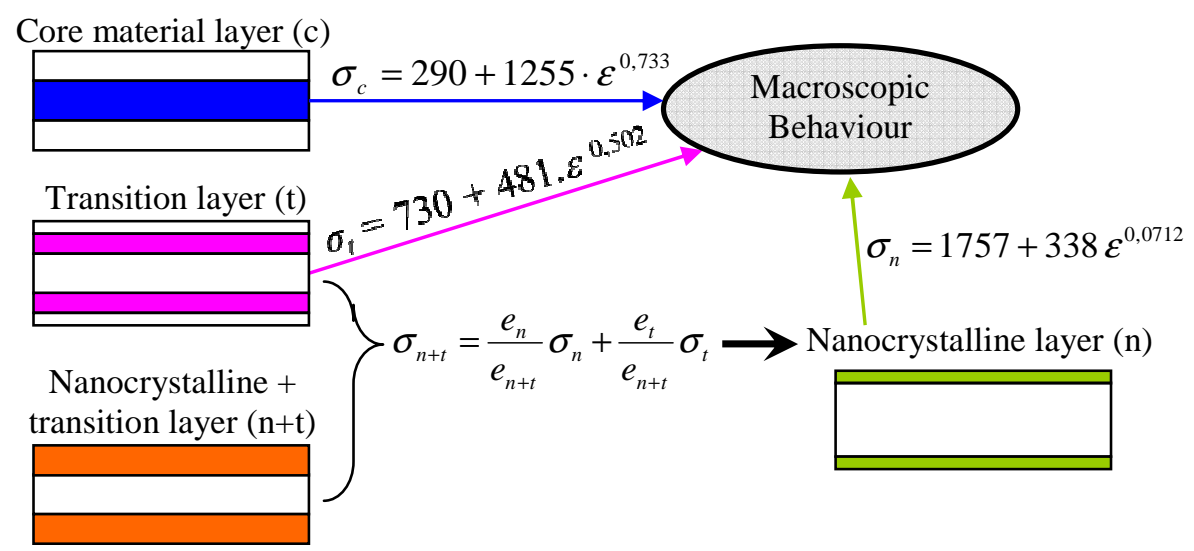

Fig. 2: Implemented approach to recover the macroscopic behaviour of the SMATed sample. 


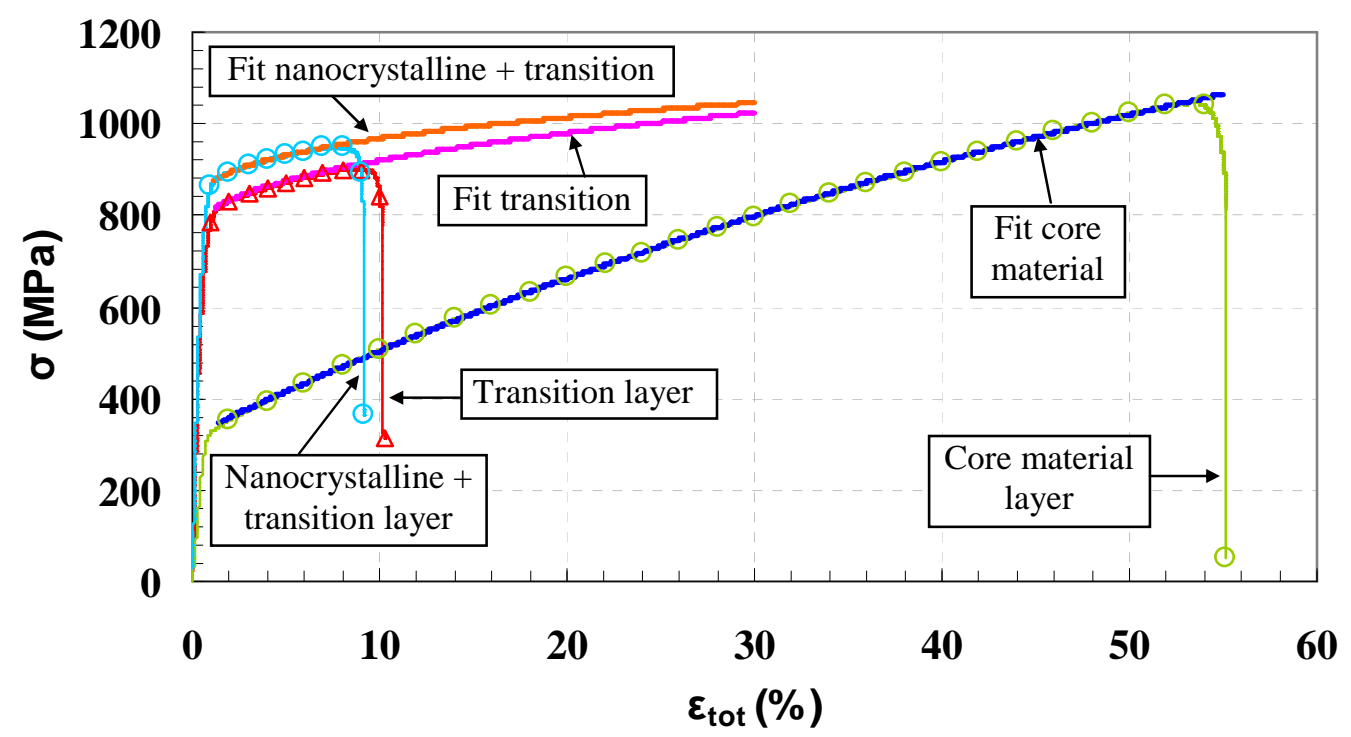

Fig. 3: True stress - true strain curves and best fits for the transition layer, the core material and the nanocrystalline + transition layers 

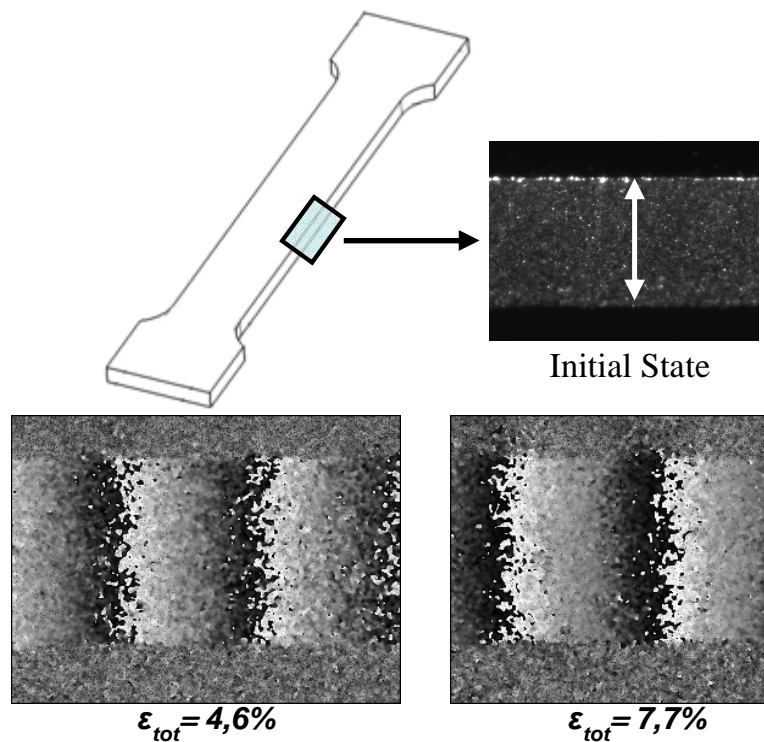

Initial State
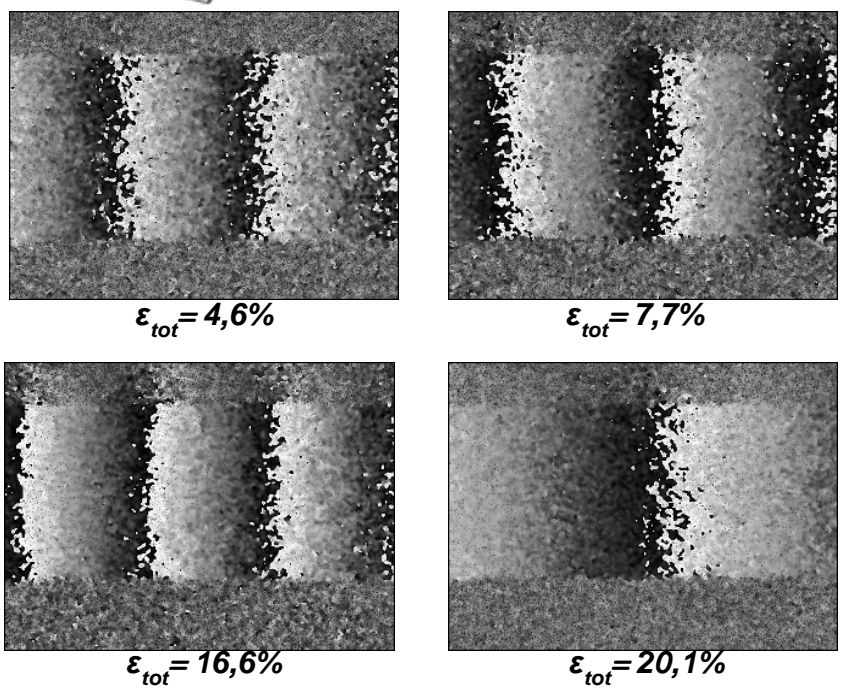

Fig. 4: Straight and parallel fringe pattern obtained by ESPI through the thickness of the specimen, confirming the hypothesis that the plane and normal to the median fibre sections remain plane and normal to the median fibre during the deformation. 


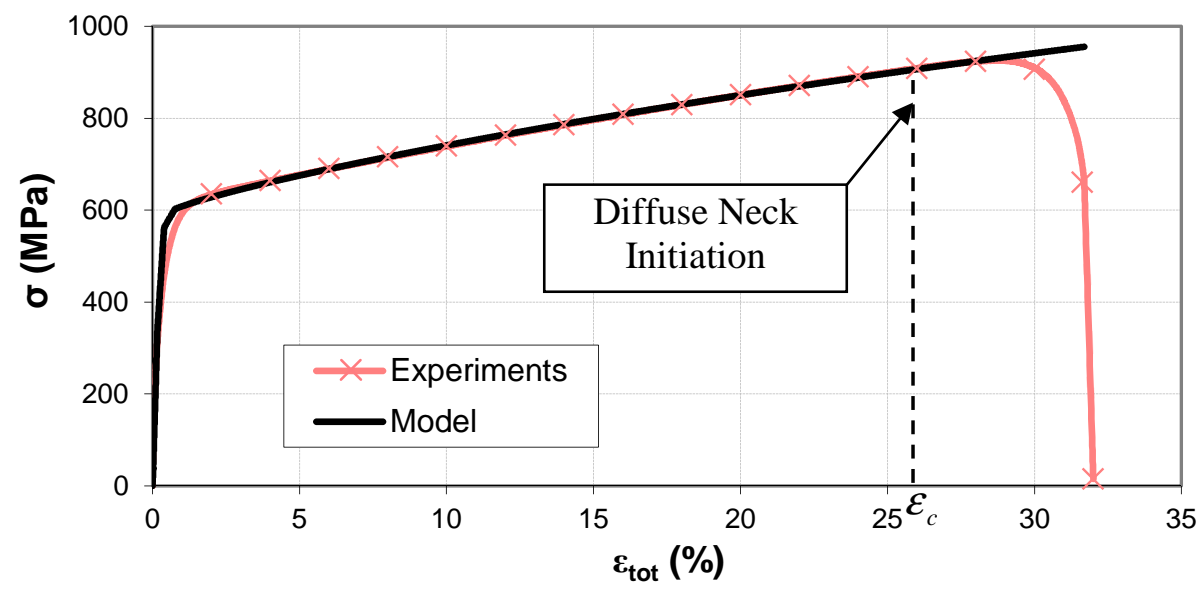

Fig. 5: True stress-true strain curve obtained experimentally and by the multilayer model of Fig. 1. 